Check for updates

Cite this: RSC Adv., 2018, 8, 12535

Received 16th February 2018

Accepted 21st March 2018

DOI: $10.1039 / \mathrm{c} 8 \mathrm{ra01482e}$

rsc.li/rsc-advances

\title{
Spontaneous catanionic vesicles formed by the interaction between an anionic $\beta$-cyclodextrins derivative and a cationic surfactant $\dagger$
}

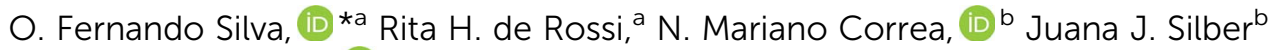 \\ and R. Darío Falcone (iD b
}

\begin{abstract}
The present work shows the synthesis of a new type of catanionic surfactant, ModCD14-BHD, which involves an anionic amphiphilic cyclodextrin and the cationic benzyl- $n$-hexadecyldimethylammonium $(B H D)$. It is obtained from the simple association of the cationic surfactant benzyl- $n$ hexadecyldimethylammonium chloride $(B H D C)$ and $\beta$-cyclodextrin $(\beta-C D)$ monosubstituted with an alkenyl succinate group (Mod- $\beta-C D 14)$. ModCD14-BHD form unilamellar vesicles spontaneously in water, while the individual components (BHDC and Mod- $\beta-C D 14)$ do not. The vesicles were characterized by dynamic light scattering (DLS), transmission electron microscopy (TEM), scanning electron microscopy (SEM) and ${ }^{1} \mathrm{H}$ NMR techniques. We suggest that the formation of an inclusion complex between some of the cyclodextrins units and the long hydrocarbon moiety of the cationic surfactant play a crucial role in the vesicles formation. Besides, some or the cavities are available to interact with an external guest. We think that the new surfactant molecule has properties that may lead to important applications in biomedical and pharmaceutical sciences.
\end{abstract}

\section{Introduction}

The construction of nanocarriers for drug delivery is an area of current scientific interest. The main reason for that is to explore new ways for deliver therapeutics agents directly into the desired zone and so decreasing the side effects of the drug in living organisms. Vesicles play an important role in this area because they can be constructed with bio-compatible materials and can be designed to have very interesting properties such as to respond to external stimuli. ${ }^{1}$

Among the compounds that form vesicles, we can mention catanionic surfactants which form different kinds of aggregates and have been subject to extensive experimental investigations. ${ }^{2-5}$ It has been demonstrated that they form molecular bilayers $^{6,7}$ and that they are good candidates as drug delivery vehicles, ${ }^{8}$ as well as in other areas such as detergents, foaming, etc. $^{9}$

\footnotetext{
${ }^{a}$ Instituto de Investigaciones en Físico-Química de Córdoba, INFIQC-CONICET, Facultad de Ciencias Químicas, Departamento de Química Orgánica, Universidad Nacional de Córdoba, Ciudad Universitaria, Córdoba, Argentina. E-mail: fersilva@ fcq.unc.edu.ar

${ }^{b}$ Departamento de Química, Universidad Nacional de Río Cuarto, Agencia Postal \# 3, C. P. X5804BYA, Río Cuarto, Argentina

$\dagger$ Electronic supplementary informations (ESI) available: Size distribution at different time, scanning electron microscopy micrographs (SEM) of ModCD14-BHD vesicles and experimental details included materials and methods, are available free of charge. See DOI: 10.1039/c8ra01482e
}

During the screening of self-assemblies systems suitable for various applications, the combination of catanionic surfactants with macrocycles have been studied. In recent years several reports involving calixarenes appeared in the literature, ${ }^{\mathbf{1 0}}$ but to the best of our knowledge, there are no reports involving cyclodextrins. It is worth to mention that a zwitterionic cyclodextrin bearing positive groups on one rim and negative groups on the other has been reported. ${ }^{11}$ This compound assembles in water forming elongated tape-like structures through the hydrogen bonding interaction of the ammonium group and the carboxylate group of adjacent molecules. ${ }^{\mathbf{1 1}}$

It is also important to remark that there are two studies using $\beta$ cyclodextrin $(\beta-\mathrm{CD})$ to induce transition phase in nonstoichiometric mixed cationic and anionic surfactants. ${ }^{12,13}$ In those reports, the transition micelle to vesicles is achieved by altering the composition of mixed surfactants due to the selective formation of inclusion complexes between $\beta$-CD and the major component in the mixed system. ${ }^{\mathbf{1 2 , 1 3}}$

In our lab, we have synthetized amphiphilic CD derivatives from the esterification of one of the $\mathrm{OH}$ groups with an alkenyl succinic acid. ${ }^{\mathbf{1 4}}$ These CDs resemble surfactants molecules and form micelles in water, monolayers at the air-aqueous interface ${ }^{15}$ and can be solubilized in reverse micelles. ${ }^{16,17}$ We considered that this type of molecules might be good candidates to form catanionic surfactants with a peculiar feature, they have an additional recognition site, namely the cavity of cyclodextrin, which might be important for the interaction of the organized structures with a desired guest. 
In this communication we present the synthesis of a new catanionic surfactant (ModCD14-BHD in Scheme 1) formed by the association of a cationic surfactant, benzyl- $n$-hexadecyldimethylammonium chloride (BHDC in Scheme 1), and an amphiphilic $\beta$-CD (Mod- $\beta$-CD14 in Scheme 1).

\section{Results and discussion}

The anion of Mod- $\beta$-CD14 (ModCD14) combines with the benzyl- $n$-hexadecyldimethylammonium (BHD) producing ModCD14-BHD which self-assemblies in water forming stable vesicles. Mod- $\beta$-CD14 is generated by reaction between 2tetradecen-1-yl succinic anhydride and $\beta$-CD as a mixture of two isomers (1b and $1 \mathrm{c}$ in Scheme 1). ${ }^{15}$ We obtained ModCD14-BHD using a $1: 1$ molar mixture of the cationic and anionic surfactants and after elimination of the counterions as is described in the ESI. $\dagger$ Our goal was to explore the behavior of ModCD14BHD in water in order to generate self-assemblies such as vesicles that integrated a macrocyclic structure large enough to play the role of a host for small molecules in their hydrophobic core. The techniques used to characterize these systems were: dynamic light scattering (DLS), transmission electron microscopy (TEM), scanning electron microscopy (SEM) and ${ }^{1} \mathrm{H}$ NMR.

When ModCD14-BHD was dissolved in water up to $5 \times$ $10^{-3} \mathrm{M}$, an opalescent solution was obtained (Fig. S1 in ESI $\dagger$ ) which was stable for at least one month. This result contrasts with the fact that neither Mod- $\beta$-CD14 nor BHDC (Scheme 1) are able to form vesicles in water.

Experiments using dynamic light scattering (DLS) showed the formation of catanionic aggregates in water with diameters ranging from $220-240 \mathrm{~nm}$ with low polydispersity index (0.20.3 ). For example, in Fig. 1A is plotted the size distributions of ModCD14-BHD vesicles in water at different surfactant concentrations. Moreover, the sizes were independent of the ModCD14-BHD concentration in the range of $1 \times 10^{-5}$ to $1 \times$ $10^{-4} \mathrm{M}$. These assemblies exhibited good stability over time as
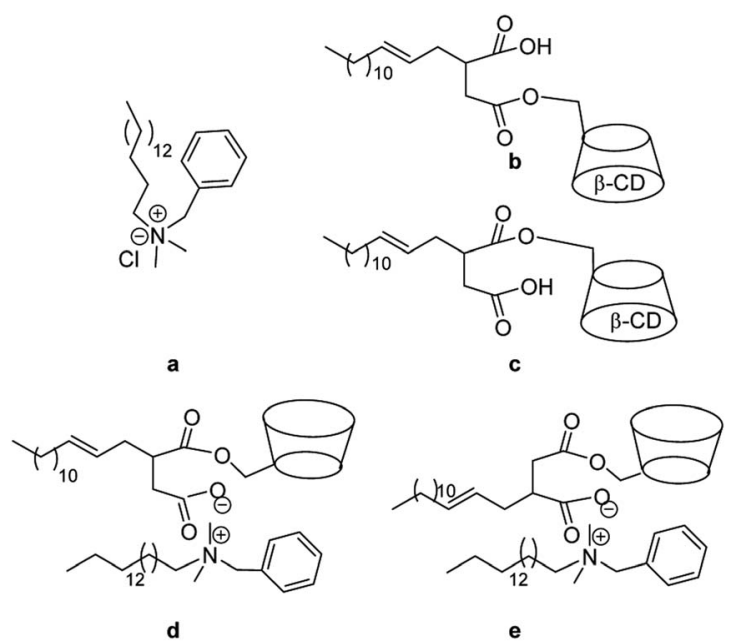

Scheme 1 Molecular structure of BHDC (a), isomers of the amphiphilic CDs (Mod- $\beta$-CD14) (b and c) and catanionic surfactant ModCD14-BHD (d and e).
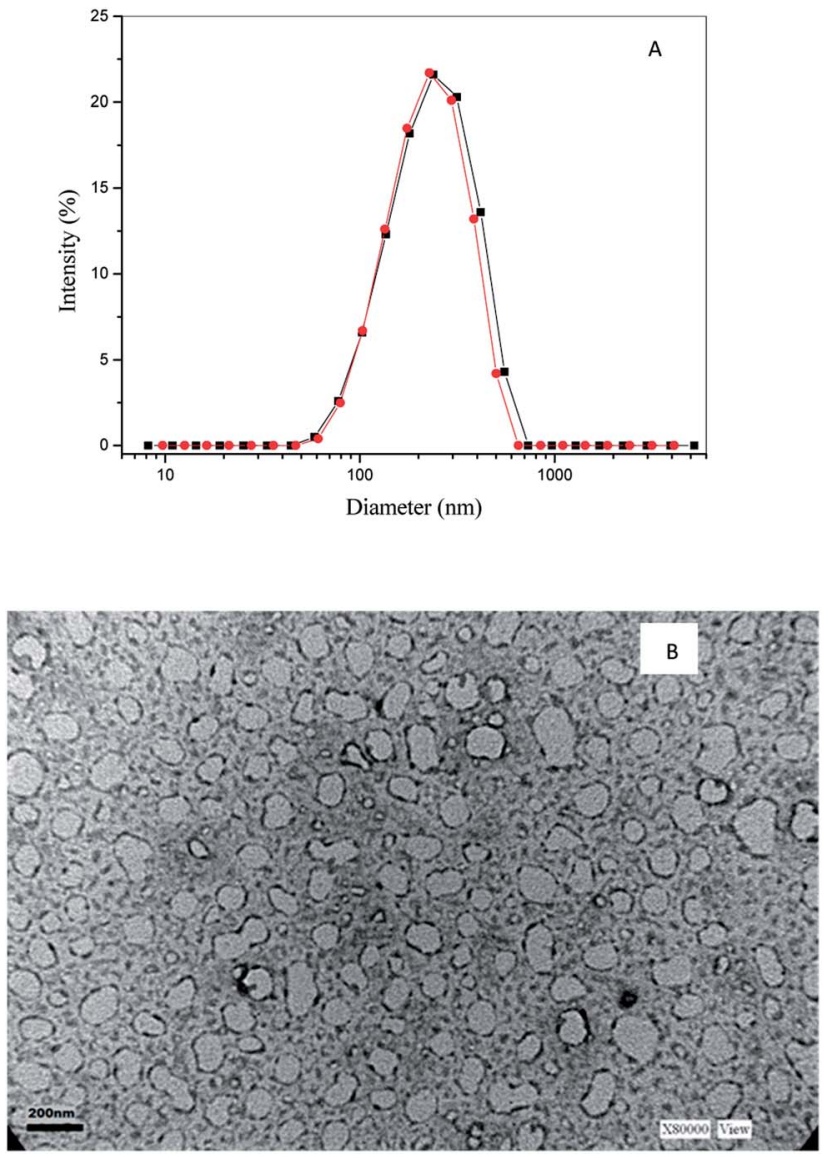

Fig. 1 (A) Size distributions of ModCD14-BHD vesicles (measured by dynamic light scattering) in water at different surfactant concentrations, $1 \times 10^{-4} \mathrm{M}$ (squares) and $8 \times 10^{-5}$ (circles). $T=25^{\circ} \mathrm{C}$. (B) TEM image (negatively stained $2 \%$ phosphotungstic acid) of ModCD14$B H D$ vesicles. [ModCD14-BHD] $=5 \times 10^{-4} \mathrm{M}$.

indicated by DLS analysis carried out two weeks after the first measure with similar diameters and polydispersity values (Table S1 in ESI $\dagger$ ).

It is well known that the size of vesicles do not change with the surfactant concentration, ${ }^{\mathbf{1 8}}$ therefore the fact that the diameter of the aggregates of [ModCD14-BHD] measure at low and high surfactant concentration is approximately the same represent a good evidence that the organized system created contain vesicles and not micelles. ${ }^{19}$

Further characterization of the system was done by transmission electron microscopy (TEM) acquiring micrographs of the solution containing ModCD14-BHD (Fig. 1). As can be observed, the diameters of the aggregates and the homogeneous distribution are comparable to those measured by DLS. Besides, these results are in concordance with those obtained by scanning electron microscopy (SEM) (Fig. S2 in ESI $\dagger$ ) The sizes of vesicles obtained from TEM and SEM (Fig. 1 and S2) were slightly smaller than those obtained from DLS analysis. The small differences in sizes can be attributed to the hydration level of the aggregates since DLS measures the hydrodynamic diameter of particles in solution while TEM and SEM values arise from direct inspection in the absence of solvent. ${ }^{20}$ 
It is well known that CDs have strong affinity for hydrocarbon chains, ${ }^{21}$ and we demonstrated previously that the alkenyl side chain grafted to the cyclodextrin is included into the CDs cavity. ${ }^{24}$ In addition the association equilibrium constants reported for $\beta$-CD and hexadecyltrimethylammonium bromide (C16TAB) are in the range of $(4.5-7.0) \times 10^{4} \mathrm{M}^{-1} .^{21}$ Therefore, it was reasonable to expect a competitive inclusion between BHD hydrocarbons with alkenyl chains for the CD cavity in the ModC14-BHD aggregates. These interactions were explored by ${ }^{1} \mathrm{H}$ NMR. Fig. 2 shows the ${ }^{1} \mathrm{H}$ NMR spectra of the CD protons region of ModCD14-BHD and Mod- $\beta$-CD14. It can be seen that the signals of $\mathrm{H}-3$ and $\mathrm{H}-5$ of the catanionic surfactant (Fig. 2A) shift upfield and $\mathrm{H}-2$ and $\mathrm{H}-4$ shift downfield in comparison with the amphiphilic cyclodextrin (Fig. 2B). The shifts of protons $\mathrm{H}-3$ and $\mathrm{H}-5$, which are located inside the cavity, suggest an inclusion process. On the other hand, protons $\mathrm{H}-2$ and $\mathrm{H}-4$ located in the outer surface of cyclodextrins are also perturbed as consequence of formation of the assemblies.

To determine the nature of the interaction we performed 2D ${ }^{1} \mathrm{H}-{ }^{1} \mathrm{H}$ NMR (NOESY) and the obtained results are shown in Fig. 3. The cross correlation peaks of the phenyl, $N$-methyl, methylene and methyl proton signals with proton signals of the sugar proton $\mathrm{H}-3$ and H-5 located in the cavity of the cyclodextrin clearly indicates inclusion of these moieties into the cyclodextrin cavity. It worth to mention that methyl or methylene signals correspond to the interaction between $\mathrm{H}-3$ and $\mathrm{H}-5$ of $\mathrm{CD}$ and the hydrocarbons chains of BHD as well as alkenyl chains linked to $\mathrm{CD}$ as was mentioned above also, we observe crossed correlation peaks between the above mentioned groups with proton $\mathrm{H}-2$ and $\mathrm{H}-4$ located in the external surface of cyclodextrins. The results indicate that some of the BHD hydrocarbon chains are included into the cavity of CD while others interact with the external wall of the macrocycle.

Taking into account all the results presented, we propose the arrangement shown in Scheme 2, which seems to be the most favorable since the hydrophilic cyclodextrin moiety is exposed to water. In a previous work, ${ }^{17}$ we have demonstrated that this kind of CDs interact with the ammonium moiety of BHDC

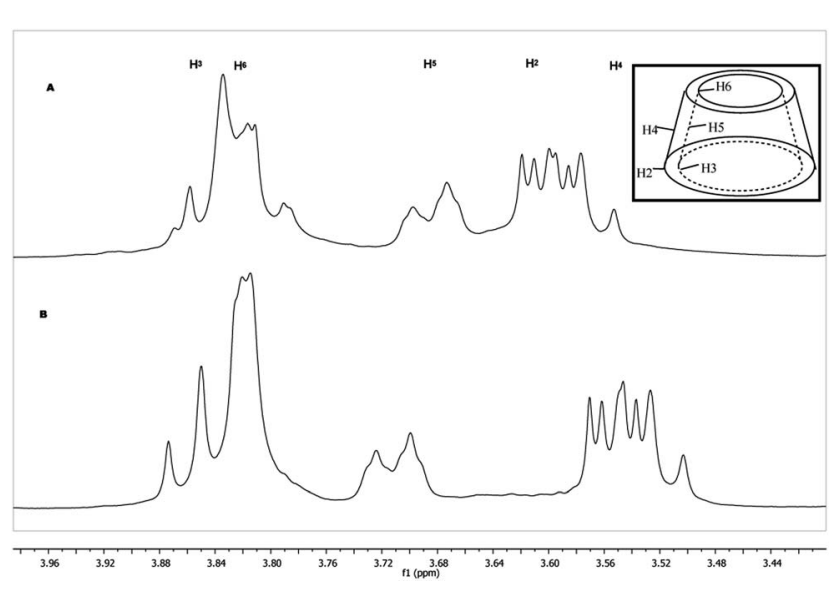

Fig. $2{ }^{1} \mathrm{H}$ NMR spectra of Mod C14-BHD catanionic surfactant $(A)$ and Mod- $\beta-C D 14$ (B) (400 MHz, $D_{2} O$ ). Expansion of the sugar protons region. Anomeric proton is not shown. [ModCD14-BHD] $=5 \times$ $10^{-4} \mathrm{M}$. Inset: schematic structure of cyclodextrins.

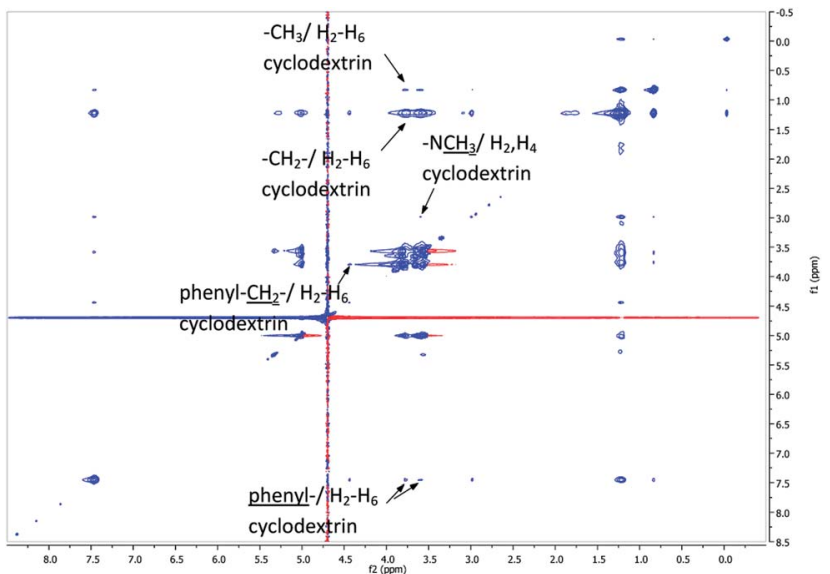

Fig. $3{ }^{1} \mathrm{H}-{ }^{1} \mathrm{H}$ 2D NOESY NMR spectrum of ModCD14-BHD $5 \times$ $10^{-4} \mathrm{M}$ in $\mathrm{D}_{2} \mathrm{O}$. The signals pointed with arrows show through-space ${ }^{1} \mathrm{H}-{ }^{1} \mathrm{H}$ interactions between phenyl, $\mathrm{N}$ methyl, methylene and methyl protons with the glucose protons of the cyclodextrin.

through its carboxylic group, hence we expected a strong ionpair interaction between the carboxylate group and ammonium moiety in ModCD14-BHD as shown in scheme 2. Furthermore, based on the data of NMR experiments, the inclusion complex with the CDs cavity could be formed with the alkyl or $N$-methyl groups of the cationic moiety. The presence of other types of inclusion complexes, i.e. those obtained by inclusion of alkenyl chains into the CDs cavity, cannot be discarded but for clarity, only one of them is shown Scheme 2 .

We believe that the formation of inclusion complexes between CDs and the hydrocarbon moieties linked to the cationic surfactant play a crucial role in the vesicles formation. As a proof of that, when a large excess of adamantylamine which is known to form strong inclusion complex with $\beta-\mathrm{CD},{ }^{22}$ was added to the catanionic surfactant, the formation of the vesicles do not take place even shaking the suspension for $72 \mathrm{~h}$.

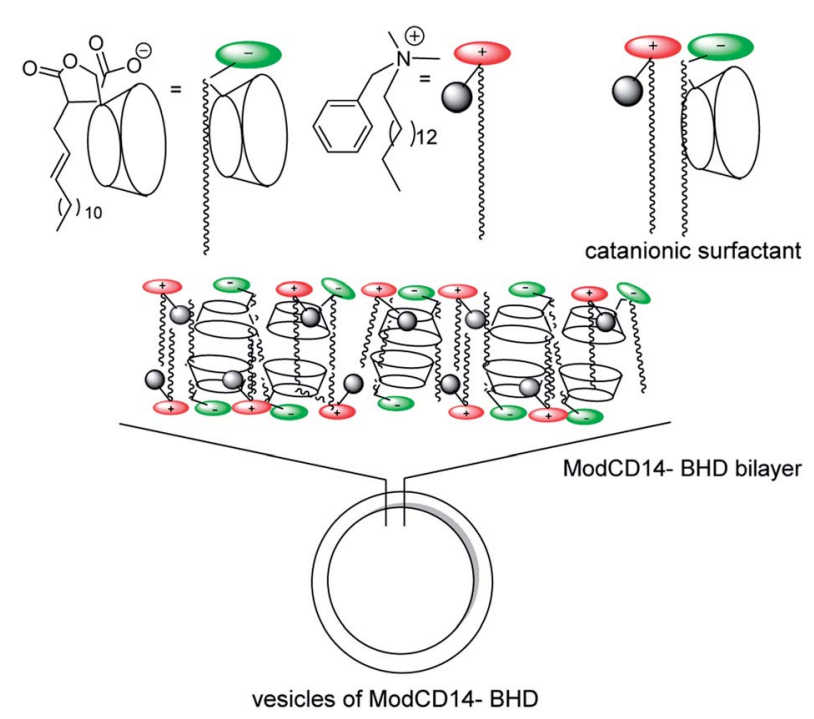

Scheme 2 Schematic representation of the catanionic surfactant and its conformation at the bilayer. 


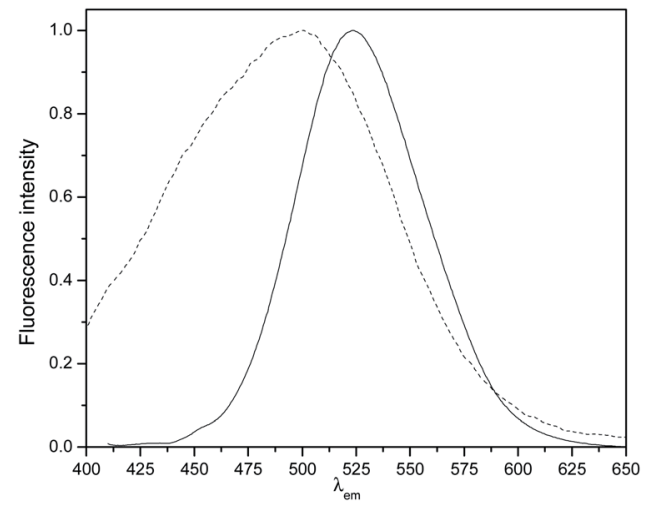

Fig. 4 Normalized fluorescence spectra of PRODAN in water (line) and in a solution of $5.0 \times 10^{-4} \mathrm{M}$ of ModCD14-BHD (dash line). $[\mathrm{PRODAN}]=2 \times 10^{-6} \mathrm{M}$.

However not all the cavities are occupied and, the vesicles are able to interact with external guests as demonstrated using PRODAN as a molecular probe. In Fig. 4, we showed the normalized fluorescence spectra of PRODAN in water and, in a solution of vesicles of ModCD14-BHD. We observed that the band around $\lambda=525 \mathrm{~nm}$, which correspond to PRODAN in water shift toward lower wavelength up to $\lambda=500 \mathrm{~nm}$ when the concentration of ModCD14-BHD is $=5.0 \times 10^{-4} \mathrm{M}$. The hypsochromic shift of the emission band indicates that PRODAN sense a less polar environment in comparison with water. ${ }^{5,23}$ The emission at $\lambda=430 \mathrm{~nm}$ is attributed to the location of PRODAN in the nonpolar region of the bilayer. ${ }^{5,23}$ The emission band which appears at $\lambda_{\max }=500 \mathrm{~nm}$ could be due to the emission of PRODAN from the cavity of cyclodextrins. ${ }^{24,25}$

To explore the availability of cyclodextrin to form complex with external molecules, we conducted measurements of induced circular dichroism (ICD) using PRODAN as a probe (Fig. 5). It is known that molecules that are not optically active generally show ICD spectra when associated with cyclodextrins due to chirality transfer. This induced optical activity arises due to perturbation in the electronic transition of the guest molecule, by the chiral field of the macrocycle. ${ }^{26}$

In Fig. 5, the ICD spectra shows a signal (positive and negative) that was interpreted suggesting that the electric transition dipole of PRODAN $^{27}$ during the absorption are

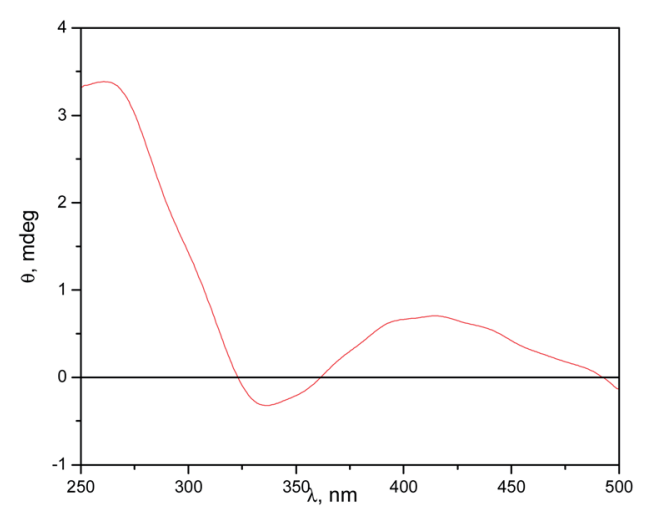

Fig. 5 Induced circular dichroism spectra of PRODAN in a solution of $5.0 \times 10^{-4} \mathrm{M}$ of ModCD14-BHD (dash line). [PRODAN] $=1 \times 10^{-4} \mathrm{M}$. parallel and perpendicular respectively to the principal axis of the cyclodextrin..$^{28}$ This result indicate that there is interaction of PRODAN with the chiral cyclodextrin molecule, although it might not be fully included into the cavity.

\section{Conclusions}

The present work shows, for the first time a new type of catanionic surfactant (ModCD14-BHD) formed by combination of modified $\beta$-CDs (Mod- $\beta$-CD14) and a cationic surfactant (BHDC), which has the principal feature to create spontaneously vesicles in water, while BHDC and Mod- $\beta$-CD14 do not. The lack of toxicity of the $\mathrm{CDs}^{29}$ and the fact that BHDC is a compound approved by the USP ${ }^{30}$ convert to the vesicles of ModCD14-BHD as potential drug carriers exploiting the possibility to load molecules in different region of the assemblies.

The use of drug delivery systems, based on vesicles created by ModCD14-BHD could represent an innovative strategy that enables effective therapy. Thus, there is a need to move a step forward and utilize an advanced technology based on combination strategy, which exploits the advantages of both the systems, namely, cyclodextrin complexation and nanotechnology in single delivery system. In this sense, we believe that the catanionic vesicles of ModCD14-BHD are an excellent choice in this field.

\section{Conflicts of interest}

There are no conflicts to declare.

\section{Acknowledgements}

Financial support from Consejo Nacional de Investigaciones Científicas y Técnicas (CONICET, PIP CONICET 112-20110100204 and 112-201101-00441), Universidad Nacional de Río Cuarto, Agencia Nacional de Promoción Científica y Técnica (PICT 2012-0232, 2012-0526, 2015-0585 and 2008-0180), and Ministerio de Industria, Comercio, Minería y Desarrollo Científico Tecnológico de Córdoba (PID-2013 and 2009-2011) is gratefully acknowledged. O. F. S., R. H. R., N. M. C., J. J. S. and R. D. F. hold a research position at CONICET. The authors thank Dr. Alejo C. Carreras from the Facultad de Matemática, Astronomía y Física (FAMAF), Universidad Nacional de Córdoba, Argentina for his help in the SEM experiments.

\section{Notes and references}

1 (a) K. Wang, D. S. Guo, X. Wang and Y. Liu, ACS Nano, 2011, 5, 2880-2894; (b) M. Baâzaoui, I. Béjaoui, R. Kalfat, N. Amdouni, S. Hbaieb and Y. Chevalier, RSC Adv., 2016, 6, 72044-72054; (c) B. G. Bag., S. Das, S. N. Hasan and A. Chandan Barai, RSC Adv., 2017, 7, 18136-18143.

2 L. Chiappisi, H. Yalcinkaya, V. K. Gopalakrishnan, M. Gradzielski and T. Zemb, Colloid Polym. Sci., 2015, 1-13.

3 C. C. Villa, F. Moyano, M. Ceolin, J. J. Silber, R. D. Falcone and N. M. Correa, Chem.-Eur. J., 2012, 18, 15598-15601. 
4 C. C. Villa, J. J. Silber, N. M. Correa and R. D. Falcone, ChemPhysChem, 2014, 15, 3097-3109.

5 C. C. Villa, N. M. Correa, J. J. Silber, F. Moyano and R. D. Falcone, Phys. Chem. Chem. Phys., 2015, 17, 1711217121.

6 J. Zhang and S. Liu, Phys. Chem. Chem. Phys., 2011, 13, 12545-12553.

7 L. Chen, H. Xing, P. Yan, J.-M. Ma and J.-X. Xiao, Soft Matter, 2011, 7, 5365-5372.

8 (a) V. V. Dhawan and M. S. Nagarsenker, J. Controlled Release, 2017, 266, 331-345; (b) D. Wang, Y. Cao, C. Chen, M. Cao, Y. Sun, J. Wang and H. Xu, RSC Adv., 2016, 6, 103224103231; (c) S. Stagnoli, M. A. Luna, C. C. Villa, F. Alustiza, A. Niebylski, F. Moyano, N. M. Correa and R. D. Falcone, RSC Adv., 2017, 7, 5372-5380.

9 A. Stocco, D. Carriere, M. Cottat and D. Langevin, Langmuir, 2010, 26, 10663-10669.

10 (a) V. Francisco, N. Basilio, L. Garcia-Rio, J. R. Leis, E. F. Maques and C. Vázquez-Vázquez, Chem. Commun., 2010, 46, 6551-6553; (b) C. Costa, V. Francisco, S. G. Silva, M. L. C. do Vale, L. García-Río and E. F. Marques, Colloids Surf., A, 2015, 480, 71-78; (c) C. Bize, J.-C. Garrigues, M. Blanzat, I. Rico-Lattes, O. Bistri, B. Colasson and O. Reinaud, Chem. Commun., 2010, 46, 586-588.

11 B. J. Ravoo, R. Darcy, A. Mazzaglia, D. Nolan and K. Gaffney, Chem. Commun., 2001, 827-828.

12 C. Dai, Z. Yang, H. Yang, Y. Liu, J. Fang, W. Chen, W. Li and M. Zhao, Colloids Surf., A, 2016, 498, 1-6.

13 L. Jiang, M. Deng, Y. Wang, D. Liang, Y. Yan and J. Huang., J. Phys. Chem. B, 2009, 113, 7498-7504.
14 C. J. González and R. H. de Rossi, ARKIVOC, 2001, 87-99.

15 R. V. Vico, O. F. Silva, R. H. de Rossi and B. Maggio, Langmuir, 2008, 24, 7867-7874.

16 O. F. Silva, J. J. Silber, R. H. de Rossi, N. M. Correa and M. A. Fernández, J. Phys. Chem. B, 2007, 111, 10703-10712.

17 O. F. Silva, N. M. Correa, J. J. Silber, R. H. de Rossi and M. A. Fernández, Langmuir, 2014, 30, 3354-3362.

18 Liposomes. A practical approach, ed. R. R. C. New, Oxford University Press Inc., New York, 1990.

19 S. Šegota and D. Težak, Adv. Colloid Interface Sci., 2006, 121, 51-75.

20 J. I. Goldstein, D. E. Newbury; P. Echlin, D. C. Joy; C. Fiori and E. Lifshin, Scanning Electron Microscopy and X-Ray Microanalysis, Plenum, New York, 2nd edn, 1992.

21 A. J. M. Valente and O. Söderman, Adv. Colloid Interface Sci., 2014, 205, 156-176.

22 H. Goto, Y. Furusho and E. Yashima, J. Am. Chem. Soc., 2007, 129, 109-112.

23 F. Moyano, M. A. Biasutti, J. J. Silber and N. M. Correa, J. Phys. Chem. B, 2006, 110, 11838-11846.

24 O. F. Silva, M. A. Fernández, S. L. Pennie, R. R. Gil and R. H. de Rossi, Langmuir, 2008, 24, 3718-3726.

25 A. Banerjee, B. Sengupta, S. Chaudhuri, K. Basu and P. K. Sengupta, J. Mol. Struct., 2006, 794, 181-189.

26 S. Allenmark, Chirality, 2003, 15, 409-422.

27 C. C. Vequi-Suplicy, K. Coutinho and M. Teresa Lamy, Phys. Chem. Chem. Phys., 2013, 15, 11800-11807.

28 M. Kodakat, J. Am. Chem. Soc., 1993, 115, 3702-3705.

29 G. Crini, Chem. Rev., 2014, 114, 10940-10975.

30 U. S. Pharmacopeia, USP35-NF30, 2012, pp. 1708-1710. 